\title{
Restoration of Lumbrical Function after Irreversible Ulnar Nerve Injury by Modified Bunnel's Procedure (A Report of Five Cases)
}

\author{
Ali Zein A.A. Alkhooly ${ }^{1 *}$ and Ahmed Ali Z.A.A. \\ Alkhooly ${ }^{2}$ \\ ${ }^{1}$ Department of Ortho and Trauma, Minia University \\ hospital, Egypt \\ ${ }^{2}$ Department of Ortho and Trauma, Matai General \\ Hospital (El-Minia), Egypt \\ *Corresponding author: Ali Zein Alabdeen Ahmed \\ Alkhooly, Department of Ortho and trauma, Minia \\ University hospital, Egypt
}

Received: February 01, 2017; Accepted: March 14, 2017; Published: March 21, 2017

\begin{abstract}
Normal hand function depends on the balance between the extrinsicintrinsic and extensor-flexor muscle groups. Despite their small size, the intrinsic muscles, contribute about $50 \%$ of grip strength. Clawing, partial or total due to ulnar nerve palsy affects greatly, hand's ability for fine movements, grip and skilful use along with loss of tactile sensation of hand, which usually precedes the motor weakness. We evaluated the functional results of Modified Bunnel's procedure in management of 5 patients of claw hand deformity. After a mean follow up of 16 months (range 12-24 months) results were excellent in $2(40 \%)$ cases, good in $3(60 \%)$. All the patients had improvement in the grip. No patient had Swan-neck deformity, flexion contracture, check-rein deformity, insufficient finger flexion, or superficial minus deformity. Despite being simple procedure, Modified Bunnel's procedure results in less adhesion, straight course for the tendons, good grip and leverage. The patients were satisfied with their hand function and cosmetic appearance.
\end{abstract}

Keywords: Claw hand; Ulnar nerve; Modified bunnels procedure

\section{Introduction}

The primary goals of tendon transfer procedures for ulnar nerve palsy are restoration of small and ring finger DIPJ flexion restoration of key pinch, correction of clawing, integration of MCPJ and IPJ flexion, and improvement in grip strength [1].

Many different tendon transfer procedures for restoration of key pinch have been described, including the use of wrist and finger extensors, finger flexors, and the brachioradialis to power adductor pollicis function. Both the ECRB (Smith) [2] and brachioradialis (Boyes) [3] are strong donor that can be used to restore key pinch, The ring or middle finger FDS can also be used to restore adductor pollicis function (Littler) [4]. The use of finger extensors such as the EDQ, the index EDC, and the EIP has also been described.

Correction of clawing requires correction of MCPJ hyperextension, the problem that initiates clawing. Procedures can be categorized as static or dynamic. If Bouvier's test is positive, static procedures may be successful. Osseous blocks on the dorsum of the metacarpal head have been described [5]. Zancolli described an MCPJ capsulodesis, in which a distally based flap of the volar plate was advanced proximally and sutured to the metacarpal neck, effectively limiting MCPJ extension [6]. Bunnell described a partial release of the A1 and A2 pulleys to allow bowstringing of the flexor tendons [7]. This results in increasing the moment arm of the flexor tendons at the MCPJ, thereby preventing MCPJ hyperextension. Static tenodesis with a tendon graft can also be performed. This type of static tendon graft effectively limits the amount of MCPJ extension [8].

Dynamic tenodesis can also be performed, as popularized by Fowler and Tsuge [9-11]. A tendon graft is looped through the extensor retinaculum at the wrist The two free ends of the tendon graft are passed through the intermetacarpal spaces into the palm, along the course of the lumbricals, and out to the fingers where they are inserted to the lateral bands. When the wrist is flexed, an active tenodesis effect occurs, resulting in MCPJ flexion and IPJ extension.

In the modified Stiles-Bunnell procedure $[7,12]$ the ring finger superficialis tendon is divided distally in the finger and retrieved into the palm. It is then split into four slips. Each slip is then passed along the path of the lumbrical, volar to the deep transverse metacarpal ligament, and back into the finger, where it is inserted on the lateral band. Burkhalter recommended inserting the tendon on the proximal phalanx instead of the lateral band, thereby preventing PIPJ hyperextension [13]. Zancolli described a "lasso" insertion, wherein the FDS is passed through the A1 pulley, then sutured back onto itself, resulting in improved MCPJ flexion while avoiding PIPJ hyperextension [6].

An insertion into the lateral band may be preferred if Bouvier's test is negative (clawing is complex), but it should be remembered that PIPJ hyperextension may occur.

Brand, Riordan, and others described the use of wrist-level motors to treat clawing and integrate finger flexion as well as augment grip strength $[11,14,15]$. The FCR, ECRL, ECRB, or brachioradialis may be used. The insertion can be into the lateral band, the proximal phalanx, or the A1 or A2 pulley. The main advantage of these tendon transfer procedures over the superficialis transfers is that they improve rather than worsen grip strength. In addition, there is no great loss of function at the level of the wrist. Also, because the superficialis tendon is preserved, the transfer can be inserted on the lateral band with less chance of developing PIPJ hyperextension. 


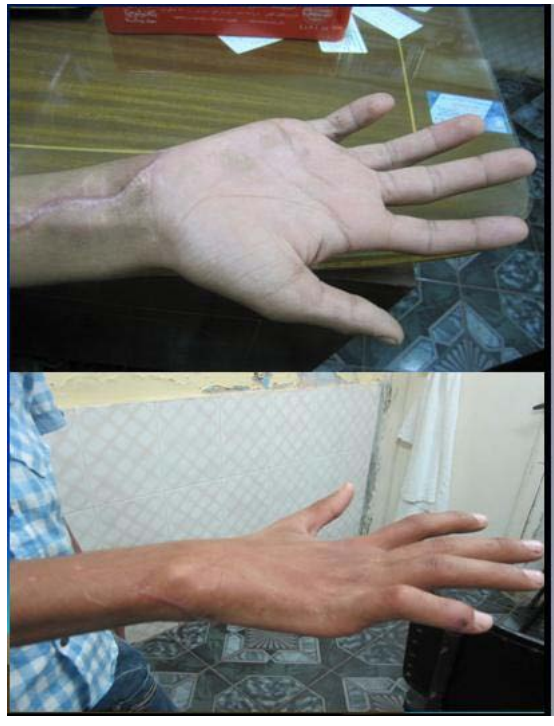

Figure 1: Pre-operative photograph of the hand.

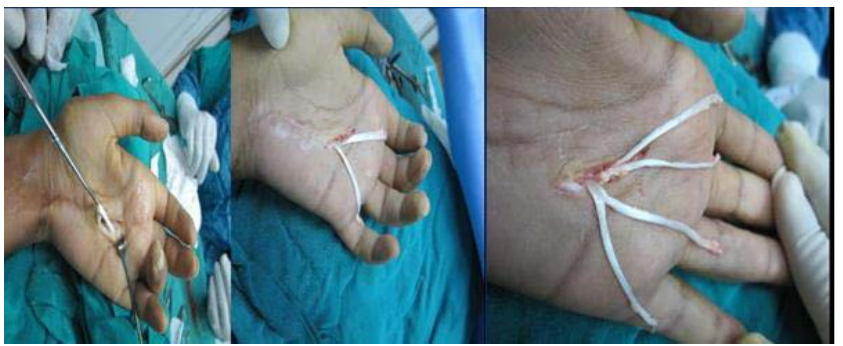

Figure 2: The ring finger's FDS was split into two and were kept wet with saline.

\section{Materials and Methods}

Five patients underwent reconstructive surgery via modified Bunnell's procedure, were included in the study. Patients were initially clinical examined and classified as per Dharmendra's classification [16]. Preoperatively, grasp was subjectively assessed by dynamometer and muscle strength charting as well as sensory function examination were done and pre-operative photograph of the hand was taken (Figure 1).

All patients were operated under general anesthesia in supine position under a pneumatic tourniquet. In cases of total clawing, an incision of $3 \mathrm{~cm}$ was made mid-laterally on radial aspect of ring finger extending from mid-shaft of proximal phalanx to mid-shaft of distal phalanx and tendon strip of Flexor Digitorum Superficialis (FDS) was detached, near to insertion as possible without injuring the Flexor Digitorum Profundus (FDP) tendon, whereas only ring finger's FDS was detached in case of isolated ulnar claw hand. Further, a 4 $\mathrm{cm}$ transverse incision was made at proximal palmar crease deep to the palmar fascia, and the slips of FDS tendons pulled out through this incision gently. In cases of the total claw hand each of the two slips were further divided to make four equal strips, whereas in ulnar clawing, the ring finger's FDS was split into two and were kept wet with saline (Figure 2).

A $2.5 \mathrm{~cm}$ longitudinal incision just lateral to mid-lateral line was

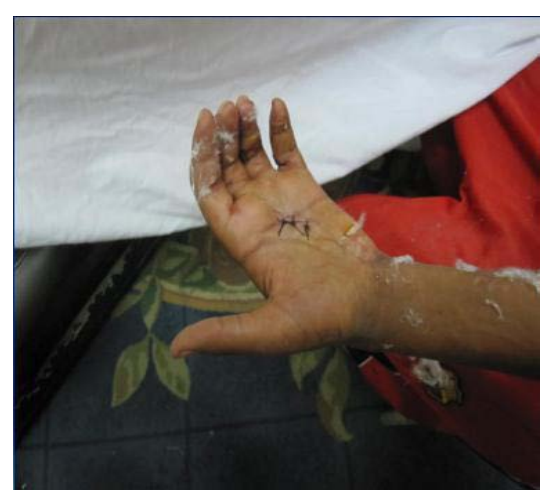

Figure 3: Sutures.

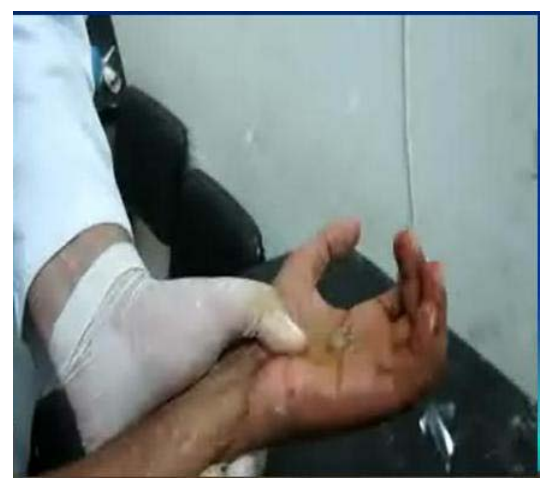

Figure 4: Follow up (Early follow up after two weeks early movement is very important).

made along the proximal segment of fingers; it was on the ulnar side whereas for middle, ring, and little it was on the radial side to secure adduction of the index finger. In each finger, extensor aponeurotic expansion was cleaned from lateral band to the edge of the middle band to avoid damage to the expansion and periosteum (Figure 2).

The tunneller was then passed through the lumbrical canal, anterior to transverse metacarpal ligament, and each slip was gently pulled into the distal incision by the tunneller. In this way, all the four tendons were pulled distally for each finger Hand was then kept on the positioning splint with $80^{\circ}$ flexion at Metacarpophalangeal Joints (MCP) joint, extension at IP joint, and $30^{\circ}$ flexion at the wrist and was tied with a sterile bandage to avoiding any displacement. The slips were then sutured with $5 / 0$ silk, a minimum of three sutures to the edge of the dorsal expansion along the axis of lumbricals, taking the slack and putting it under no tension. After burying the suture ends in all the four fingers, the tourniquet was released and bleeders caught and ligated. Layered closure was done without overlap and tension (Figure 3).

Postoperatively, below elbow slab was given as described, keeping hand in lumbrical position. The finger was left open dorsally, but slab extended right to the tip of fingers ventrally. Intravenous antibiotics were given for 3 days followed by oral antibiotics for 5 days.

Suture removal was done after 2 weeks and slab was removed after 3 weeks. In $1^{\text {st }}$ week after slab removal, elbow, wrist and MCP joint, flexion and extension exercise began without flexing the IP joint and a finger extension splint for each finger with IP joint in full extension. 


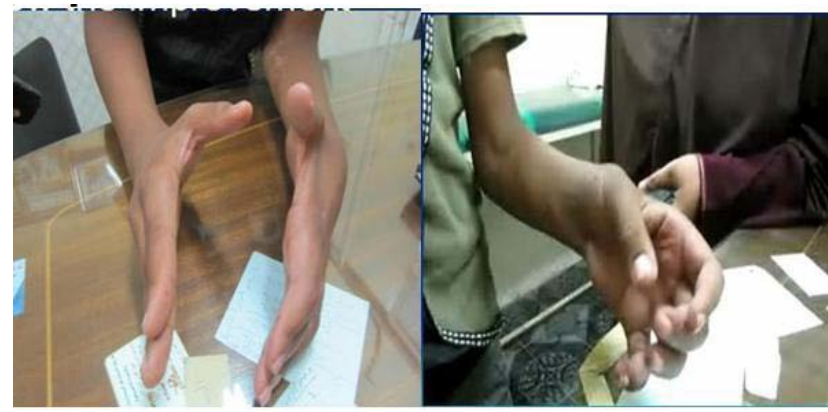

Figure 5: Follow up [extension of M P; IF joints], follow up [flexion of MP IF joints]. (Follow up after ten weeks, healthy side (left) and operated side (right)).

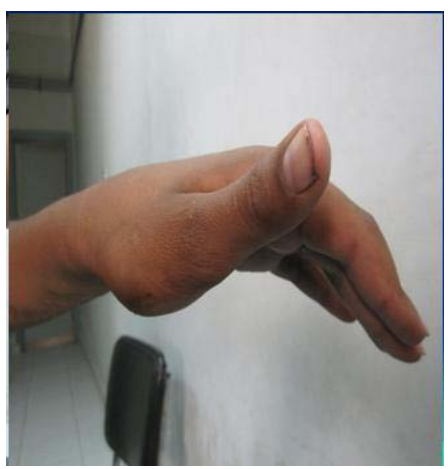

Figure 6: Flexion of MP joints and extension of interphalangeal joints.

Next week patient was encouraged lumbrical action, by contracting the transplanted muscle. After $3^{\text {rd }}$ week, patient encouraged to flex PIP joint along with wax bath and exercises. The grasp were assessed postoperatively. Results were graded as per scoring system by brand [17].

\section{Results}

Five claw hands were included in the study of which there were 4 males and one female. Average age was 25.3 years (range 19-40 years). Left hand involvement was seen in 3 cases whereas 2 had right hand involvement and no bilateral involvement. All the patients had partial claw hand (ulnar nerve). Mean follow-up was 16 months (range 1224 months). (Figure 4-7).

Duration of affection was 2 years in 3 cases, whereas 2 had more than 2 years duration. Mean duration of affection was 3.3 years (range $2-5$ years).

As per brand scoring system [18] the results were excellent 2 (40\%) cases, good in $3(60 \%)$. No fair or poor results.

The preoperative weak grasp had improved to firm grasp after surgery. Also, the hand grip improved in all patients postoperatively (Figure 8).

All the patients had improvement in the grip. No patient had Swan-neck deformity, flexion contracture, check-rein deformity, insufficient finger flexion, or superficial minus deformity. Despite being simple procedure, Modified Bunnel's procedure results in less adhesion, straight course for the tendons, good grip and leverage.

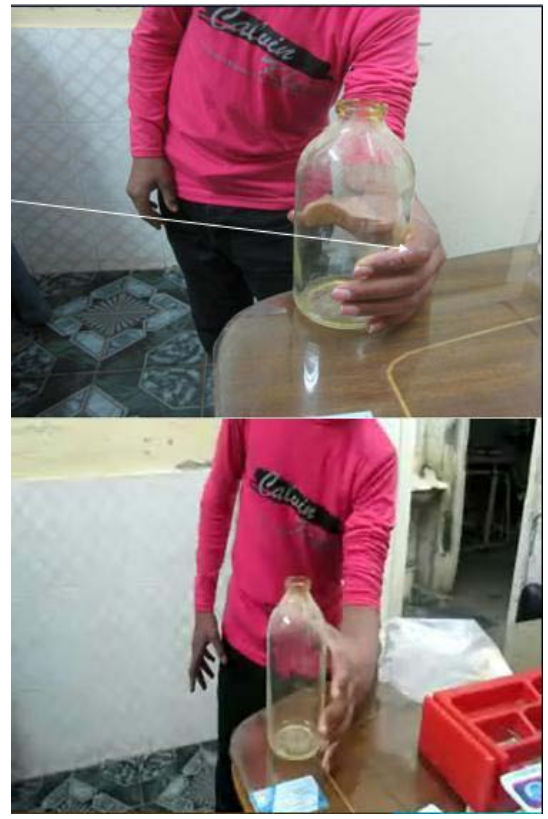

Figure 7: Gripping of large object after operation and follow up.
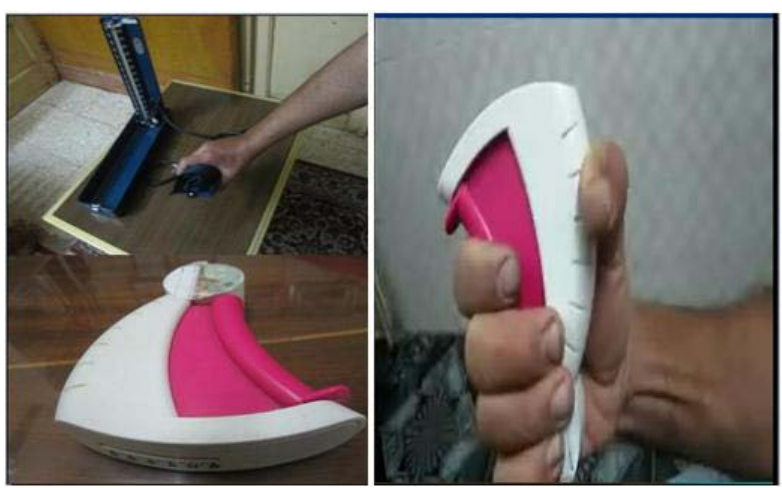

Figure 8: Simple techniques for evaluation and comparing hand grip in healthy and affected side.

The patients were satisfied with their hand function and cosmetic appearance.

\section{Discussion}

Clawing of hand, which affects the grip, the appearance of hand and ability to hold objects, are very common in ulnar nerve injury. Higher the grade, the remote were the results. Most commonly little finger had some degree of contracture probably because it was most neglected during physiotherapy and while using hand, lateral three fingers are more frequently and forcibly used than the little finger. Other causes of contracture leading to poor results are capsule contracture, disuse, dryness of non-sweating skin or skin maceration.

Surgeries to correct claw hand are based on the principle that the long finger extensors can extend IP joints, provided that hyperextension of the MCP is prevented. This stabilization of MCP can be done by capsuloplasty [15] tenodesis [11], bone block [5], arthrodesis, or tendon transfers that actively extend the IP joints and flex the MCP. The typical indication for the bone block, 
capsule plication or tenodesis is long-standing lesions with fibrosis and in muscular paralysis of central origin rather than peripheral nerve lesions. Though these are simpler procedures with success in correcting claw deformity and increasing synchronous movement between fingers when no muscle is available for transfer, they do not improve the grip strength and needs active and timely physiotherapy and exercises. Further, these are passive procedures that can never produce the active phase of intrinsic action in grasp and pinch, as they do not provide a prime flexor of MCP. Strong un-paralyzed muscles, which can be easily used for intrinsic muscle replacement by tendon transfer adding a positive voluntary action. So, if suitable muscles are available, tendon transfers should be the choice for restoration of grip strength. Littlers, Fowlers, and Brands procedures are indicated when patient is cooperative, seek earlier advice, and joints are quite supple, but are met with unsatisfactory results because of tendency for reversal of metacarpal arch due to dorsal pull and they needs very cooperative patients, who are ready for extensive physiotherapy, else potential power is dissipated resulting in useless hand. Hence, these procedures are complicated by the difficulty in rehabilitation and reports of median nerve compression due to crowding of the carpal tunnel [16].

In our patients, with low economic status, presenting deformity for a longer duration, seeking advice late with stiff joints, we agree with [17] in that, modified Bunnell's procedure is more suitable operation which has the advantage of retaining flexor superficialis tendons of the other fingers for better power grip and the avoiding swan neck deformity. Also, modified Bunnell's procedure removes the powerful flexor of the PIP joints and converted it, into an extensor of the same joints as finger flexors and the wrist flexors and extensors are strong.

Many studies Bauer B, et al. [18] reported near $80 \%$ excellent to good results, while in our study, all the 5 patients got excellent to good results. Ozkan T, et al. [19] reports Bunnell's procedure more effective in correcting claw hand deformity in comparison to zancolli lasso's procedure. Poor results were due to less cooperative patients, extensor tendon laxity, joint stiffness, and patients returning to work earlier,

Reported complications due to Bunnell's transfer are: Swan-neck deformity, flexion posture of the distal interphalangeal joint, checkrein deformity or flexion contracture, intrinsic plus deformity, and insufficient finger flexion. None of them were encountered in our cases. None of our patients developed intrinsic plus, swan-neck deformity, check-rein deformity or flexion contracture hand because as our follow-up is short and transposition was not done in mobile joints. A longer follow-up of these cases is needed to rule out these complications. Also, no case got infection or recurrence of deformity.

Proper tensioning of the tendon slips sutured at the new insertion sites is critical for a better outcome [19] among many methods suggested for equal and balanced tensioning of four tendon slip when the hand is in neutral position with respect to adduction or abduction of the wrist. It is the experience of the operating surgeon that helps to decide as to the tension that is to be kept on each slip so that maximum deformity correction is obtained without compromising the functional capabilities of the hand.
The disadvantage of taking the flexor digitorum sublimis tendon is weakening in the grasp and contributing to the position of the acute tip flexion position. Reason being that superficialis is the flexor of PIP joint and it is converted to act as extensor, thus the two muscles (superficialis and profundus) instead of acting synergically, act in opposite direction to each other.

Overall modified Bunnell's procedure is simple to perform and gives superior results as it gives least possible adhesions, provides a straight course for the tendon, and good leverage with a normal angle of approach. After surgery is it easy to re-educate the patient with better cosmetic results. The patient gets much more satisfaction as far as the functional improvement is concerned after this operation and mentally more satisfied. As all the intrinsic actions can be achieved by using FDS for all four fingers, which are attached on radial as well ulnar side to adduct and abduct as well. The only prerequisites for the Bunnell's procedure include availability of FDS along with working FDP.

The mean limitation of this study was the small number of patients and the short follow up period (Figure 4-8).

\section{Conclusions}

Patient selection, preoperative and postoperative physiotherapy, and following the principles laid by Bunnell's procedure along with proper tensioning and surgeon's experience are essential for successful treatment of clawing and obtaining better functional and cosmetic results.

\section{References}

1. Sammer DM, Chung KC. Tendon Transfers Part II: Transfers for Ulnar Nerve Palsy and Median Nerve Plast Reconstr Surg. 2009; 124: 212e-221e.

2. Smith RJ. Extensor carpi radialis brevis tendon transfer for thumb adduction-a study of power pinch. J Hand Surg Am. 1983; 8: 4-15.

3. Boyes JH. Bunnell's surgery of the hand. $5^{\text {th }}$ ed. Philadelphia: J.B. Lippincott Company; 1970.

4. Hamlin C, Littler JW. Restoration of power pinch. J Hand Surg Am. 1980; 5 : 396-401.

5. Mikhail IK. Bone Block Operation for Clawhand. Surg Gynecol Obstet. 1964; 118: $1077-1079$.

6. Zancolli EA. Claw-hand caused by paralysis of the intrinsic muscles: a simple surgical procedure for its correction. J Bone Joint Surg Am. 1957; 39: 10761080.

7. Bunnell S. Surgery of the intrinsic muscles of the hand other than those producing opposition of the thumb. Journal of Bone and Joint Surgery. 1942; 24: 1-3.

8. Parkes A. Paralytic claw fingers--a graft tenodesis operation. Hand. 1973 5: 192-199.

9. Fowler SB. Extensor apparatus of the digits. Journal of Bone and Joint Surgery. 1949; 31: 477.

10. Tsuge $\mathrm{K}$. Tendon transfers in median and ulnar nerve paralysis. Hiroshima $J$ Med Sci. 1967; 16: 29-48.

11. Riordan DC. Tendon transplantations in median-nerve and ulnar-nerve paralysis. J Bone Joint Surg Am. 1953; 35: 312-320.

12. Stiles HJ, Forrester-Brown MF. Treatment of Injuries of Peripheral Spinal Nerves. H. Frowde and Hodder and Stoughton; 1922.

13. Burkhalter WE. Restoration of power grip in ulnar nerve paralysis. Orthop Clin North Am. 1974; 5: 289-303. 
14. Brand PW. Paralytic claw hand with special reference to paralysis in leprosy and treatment by sublimis transfer of Stiles and Bunnell. J Bone Joint Surg Br. 1958; 40: 618-632.

15. Zancolli E. Intrinsic paralysis of the ulnar nerve Dphysiopathology of the claw hand. Structural and dynamic bases of hand surgery ( $2^{\text {nd }}$ edn $)$. Lippincott, Philadelphia: 1979; 159-206.)

16. Malaviya GN. Median nerve palsy following claw finger correction in leprosy: Effect of M. leprae or a consequence of surgery. Indian J Lepr. 2002; 74: 217-220
17. Calandruccio JH, Jobe MT. Paralytic hand. In: Canale ST, Beaty JH Campbell's Operative Othropaedics. $11^{\text {th }}$ edn. Philadelphia: Mosby Elsevier; 2008; 4125-4172.

18. Bauer B, Khoa NK, Chabaud B, Chaise F, Quang HT, Comtet JJ. Flexor digitorum superficialis tendon transfer for intrinsic paralysis in leprosy. Chir Main. 2007; 26: 136-140.

19. Ozkan T, Ozer K, Yukse A, Gulgonen A. Surgical reconstruction of irreversible ulnar nerve paralysis in leprosy. Lepr Rev 2003; 74: 53-62.
Austin J Orthopade \& Rheumatol - Volume 4 Issue 1 - 2017

ISSN: 2472-369X | www.austinpublishing group.com

Alkhooly et al. (@) All rights are reserved
Citation: Alkhooly AZAA and Alkhooly AAZAA. Restoration of Lumbrical Function after Irreversible Ulnar Nerve Injury by Modified Bunnel's Procedure (A Report of Five Cases). Austin J Orthopade \& Rheumatol. 2017; 4(1): 1050. 